\title{
AUTOPERCEPÇÃO DE HIPERTENSOS ACOMPANHADOS PELA ESTRATÉGIA SAÚDE DA FAMÍLIA ACERCA DA QUALIDADE DE VIDA
}

SELF-PERCEPTION OF HYPERTENSIVE PATIENTS ACCOMPANIED BY THE FAMILY HEALTH STRATEGY ON QUALITY OF LIFE

AUTOPERCEPCIÓN DE PACIENTES HIPERTENSOS ACOMPAÑADA DE LA ESTRATEGIA DE SALUD FAMILIAR SOBRE CALIDAD DE VIDA

\author{
Paloma de Vasconcelos Rodrigues ${ }^{1}$ \\ Maria Socorro de Araújo Dias ${ }^{2}$ \\ Maria da Conceição Coelho Brito ${ }^{3}$ \\ Marina Pereira Moita ${ }^{4}$ \\ Lielma Carla das Chagas Silva ${ }^{5}$
}

Andrea Carvalho Araújo Moreira 6

Palavras-chave:

Atenção Primária à Saúde; Qualidade de vida; Hipertensão.

\section{Keywords:}

Primary Health Care; Quality of Life; Hypertension

Palabras clave: Atención Primaria de Salud; Calidad de Vida; Hipertensión

Submetido: 25/09/2019

Aprovado: 13/11/2019

Autor(a) para Correspondência: Paloma de Vasconcelos Rodrigues

Escola de Saúde Pública

Visconde de Saboia Rua Maestro José Pedro, 183, centro, Sobral, Ceará. CEP: 62010-260

E-mail:palomavasconcelos@ hotmail.com

\section{RESUMO}

o estudo objetiva analisar a autopercepção da qualidade de vida de hipertensos acompanhados pela Estratégia Saúde da Família a partir do questionário SF-36. Trata-se de um recorte de uma pesquisa multicêntrica intitulada "Efetividade do Núcleo de Apoio à Saúde da Família frente ao controle clínico e qualidade de vida de hipertensos e diabéticos", aprovada pelo Programa de Pesquisa para o SUS, edital $n^{\circ}$ 01/2017. Trata-se de um estudo transversal, de abordagem quantitativa. 0 universo do estudo correspondeu ao município de Sobral. Para tabulação dos dados, optouse pelo uso do Excel. Este estudo respeitou a Resolução $n^{\circ}$ 466/2012. Participaram do estudo 125 pessoas portadoras de HAS assistidas por equipes dos Centros de Saúde da Família (CSF). Destas, 81 (65\%) eram do sexo feminino e 44 (35\%) eram do sexo masculino. É possivel observar que 80 participantes (64\%) tinham mais de 60 anos. 0 domínio da qualidade de vida mais comprometido entre os participantes do estudo foi limitação por aspecto físico, com média de 45,2. Já o dominio com maior média foi aspectos sociais $(71,4)$. Este estudo possibilitou mensurar os impactos da HAS na qualidade de vida, nas atividades de vida diária e nas relações sociais.

\footnotetext{
1. Enfermeira. Escola de Saúde Pública Visconde de Saboia. E-mail: palomavasconcelos@hotmail.com. Orcid: https://orcid.org/0000-0003-0066-1485

2. Enfermeira. Doutora em Enfermagem. Universidade Estadual Vale do Acaraú. E-mail: socorroad@gmail.com. Orcid: https://orcid.org/0000-0002-7813-547X

3. Enfermeira. Mestre em Saúde da Família. Escola de Saúde Pública Visconde de Saboia. E-mail: marycey@ hotmail.com. Orcid: https://orcid.org/0000-0002-3484-9876

4. Acadêmica de Enfermagem pela Universidade Estadual Vale do Acaraú. E-mail: marymoita28@gmail.com. Orcid: https://orcid.org/0000-0002-1920-480X

5. Enfermeira. Mestre em Saúde da Família. Escola de Saúde Pública Visconde de Saboia. E-mail: lielma_carla@ yahoo.com.br. Orcid: https://orcid.org/0000-0002-2688-9309

6. Enfermeira. Doutora em Enfermagem. Universidade Estadual Vale do Acaraú. E-mail: dreamoreira@yahoo. com.br. Orcid: https://orcid.org/0000-0001-9855-1449
} 


\section{ABSTRACT}

The study aims to analyze the self-perception of quality of life of hypertensive patients followed by the Family Health Strategy from the SF-36 questionnaire. This is a clipping from a multicenter research entitled "Effectiveness of the Family Health Support Center regarding the clinical control and quality of life of hypertensive and diabetic patients", approved by the SUS Research Program, edict 01/2017. This is a cross-sectional study with a quantitative approach. The study universe corresponded to the municipality of Sobral. For data tabulation, we chose to use Excel. This study complied with Resolution $n^{\circ} 466 / 2012$. The study included 125 people with hypertension assisted by teams from the Family Health Centers (CSF). Of these, 81 (65\%) were female and 44 (35\%) were male. 80 participants (64\%) were over 60 years old. The most compromised domain of quality of life among study participants was limitation by physical aspect, with an average of 45.2. The domain with the highest average was social aspects (71.4). This study made it possible to measure the impacts of hypertension on quality of life, daily living activities and social relationships.

\section{RESUMEN}

El estudio tiene como objetivo analizar la autopercepción de la calidad de vida de los pacientes hipertensos, seguida de la Estrategia de salud familiar del cuestionario SF-36. Este es un recorte de una investigación multicéntrica titulada "Eficacia del Centro de Apoyo a la Salud Familiar con respecto al control clínico y la calidad de vida de pacientes hipertensos y diabéticos", aprobado por el Programa de Investigación del SUS, edicto 01/2017. Este es un estudio transversal con un enfoque cuantitativo. El universo de estudio correspondió al municipio de Sobral. Para la tabulación de datos, elegimos usar Excel. Este estudio cumplió con la Resolución n 466/2012. El estudio incluyó a 125 personas con hipertensión asistidas por equipos de los Centros de Salud Familiar (CSF). De estos, 81 (65\%) eran mujeres y 44 (35\%) eran hombres. 80 participantes (64\%) tenían más de 60 años. El dominio de calidad de vida más comprometido entre los participantes del estudio fue la limitación por aspecto físico, con un promedio de 45,2. El dominio con el promedio más alto fue el de los aspectos sociales (71.4). Este estudio permitió medir los impactos de la hipertensión en la calidad de vida, las actividades de la vida diaria y las relaciones sociales.

\section{INTRODUÇÃ 0}

A Hipertensão Arterial Sistêmica é considerada um problema de saúde pública, um reflexo do atual perfil epidemiológico do Brasil. Parte desse perfil decorre da transição demográfica, com o acelerado envelhecimento da população ${ }^{1}$.

A HAS é doença crônica não transmissivel (DCNT) de maior prevalência na população brasileira, levando à diminuição da expectativa de vida da população e contribuindo significativamente para modificações na qualidade de vida das pessoas por interferir na capacidade física, emocional, interação social, atividade intelectual, exercício profissional e outras atividades do cotidiano ${ }^{2}$.

Qualidade de vida é definida como a percepção do indivíduo acerca de sua posição na vida, nos contextos culturais e sociais nos quais ele vive e em relação aos seus objetivos, expectativas, padrões e preocupações. 0 contexto incorpora a saúde física, o estado psicológico, o nível de independência, as relações sociais, as crenças pessoais, relação com aspectos do meio ambiente e pode ser influenciada, direta ou indiretamente, por fatores diversos associados à doenças ${ }^{3}$. Dessa forma, podese observar que o conceito de qualidade de vida está intrisicamente ligado a autopercepção que o indíviduo tem de sua própria saúde.

0 acompanhamento da qualidade de vida de pessoas com doenças crônicas é fundamental no planejamento de intervenções nos serviços de saúde, visto que fornece informações importantes sobre o bem estar e a prioridade dos usuários. Esse monitoramento subsidia a realização de programas de saúde, a implementação de ações efetivas no contexto da Atenção Primária à Saúde (APS) e proporciona melhor qualidade de vida aos usuários ${ }^{4}$.

0 controle da HAS é considerado um indicador fundamental para a qualidade da APS, uma vez que o hipertenso necessita de acompanhamento contínuo, permanente, longitudinal e precisa mudar seus hábitos de vida, o que requer orientação, acompanhamento profissional e educação na saúde ${ }^{5}$.

Estratégias vêm sendo utilizadas para otimizar 
- atendimento e acompanhamento de pacientes hipertensos na APS. No cenário mundial, o uso de protocolos para o acompanhamento e controle da HAS tem sido destaque ${ }^{6}$.

A HAS é um grande desafio para APS, pois é uma condição que coexiste com determinantes biológicos e socioculturais, e a abordagem a essa patologia para ser considerada efetiva exige o protagonismo dos indivíduos, famílias e comunidade ${ }^{7}$. Neste sentido, é necessário compreender que a resolutividade da atenção às pessoas hipertensas transcende 0 tratamento terapêtico e deve considerar a pessoa em sua totalidade.

Acredita-se que a Hipertensão Arterial Sistêmica é capaz de prejudicar a qualidade de vida de pessoas portadoras dessa doença crônica, dessa forma, o estudo objetiva analisar a autopercepção da qualidade de vida de hipertensos acompanhados pela Estratégia Saúde da Família a partir do questionário SF-36.

\section{METODOLOGIA}

Trata-se de um recorte de uma pesquisa multicêntrica intitulada "Efetividade do Núcleo de Apoio à Saúde da Família frente ao controle clínico e qualidade de vida de hipertensos e diabéticos", aprovada pelo Programa de Pesquisa para o SUS (PPSUS), edital 01/2017.

0 universo do estudo correspondeu a uma macrorregião de saúde do estado do Ceará, representada pelo pólo municipal de Sobral. 0 município apresenta um sistema de saúde organizado nos seguintes níveis de atenção: atenção primária, secundária e terciária. A Atenção Primária à Saúde é reconhecida nacionalmente por sua organização e desde sua implantação vem obtendo grandes avanços nesta área, tendo a Estratégia Saúde da Família (ESF) como referência de orientação do nível primário.

Apresenta nível de Índice de Desenvolvimento Humano por Município (IDHM) médio de 0,7, variando entre 0,6694 a 0,754 . 0 IDHM referente a longevidade esperado para o ano de 2010 foi de 0,8 , variando de 0,810 a 0,832 . Este valor representa um índice elevado e indicativo de uma população idosa e propensa ao desenvolvimento de adoecimentos crônicos ${ }^{8}$.

0 cálculo amostral e o processo de definição dos participantes iniciaram-se com a identificação do quantitativo de pessoas com hipertensão cadastradas no E-SUS no município de Sobral, e por CSF, junto à

\section{... Hipertensão Arterial \\ Sistêmica é capaz de prejudicar a qualidade de vida de pessoas...}

Secretaria da Saúde. Foram identificadas, no E-SUS, 16.343 pessoas com hipertensão no município de Sobral, em 2018.

Seguidamente, selecionaram-se os dois territórios com quantitativos mais expressivos de hipertensos do município. A partir desses dados, foi realizado o cálculo amostral e obtido um valor de 194 participantes, que correspondiam ao perfil investigado no estudo.

Dessa forma, após aplicação dos critérios de exclusão, participaram do estudo 125 pessoas com Hipertensão Arterial Sistêmica (HAS), assistidas por equipes dos Centros de Saúde da Família (CSF).

Para a avaliação da qualidade de vida foi utilizado o instrumento SF-36 em sua versão validada para o Brasil. 0 referido instrumento é composto por 36 itens que avaliam as seguintes dimensões da qualidade de vida: capacidade funcional (desempenho nas atividades relacionadas a o dia a dia); limitação por aspectos físicos (impacto da saúde física na vida da pessoa); dor (nível de dor e o impacto no desempenho das atividades diárias e/ou profissionais); estado geral de saúde (percepção subjetiva do estado geral de saúde); vitalidade (percepção subjetiva do estado de saúde); limitação por aspectos sociais (reflexo da condição de saúde física nas atividades sociais); limitação por aspectos emocionais (reflexo das condições emocionais no desempenho das atividades diárias e ou profissionais); e saúde mental (escala de humor e bem-estar) ${ }^{9}$.

Para tabulação dos dados, optou-se pelo uso do Excel. Foram utilizadas distintas técnicas de análise, de acordo com a natureza dos dados coletados. A análise dos escores do questionário SF-36 foi realizada a partir da pontuação de cada resposta do questionário e realização do cálculo de Raw Scale para cada domínio. Cada domínio foi avaliado em uma escala de 0 a 100 , onde zero corresponde a um pior estado de saúde e 100, a um melhor, analisando cada dimensão separadamente ${ }^{9}$.

Este estudo foi conduzido em conformidade com 
a Resolução N 466/2012 e todas suas complementares do CNS/MS. Apreciado e aprovado no Comitê de Ética em Pesquisa da Universidade Estadual Vale do Acaraú (UVA), Sob o Parecer No 2.102.876. 0s indivíduos foram esclarecidos quanto aos objetivos da pesquisa e mediante autorização para coleta de dados, assinaram o Termo de Consentimento Livre e Esclarecido (TCLE).

\section{RESULTADOS E DISCUSSÃO}

Fizeram parte desse estudo 125 participantes adultos diagnosticados com Hipertensão Arterial Sistêmica acompanhados pela Atenção Primária à Saúde. Com relação ao sexo dos participantes, 81 (65\%) eram do sexo feminino e $44(35 \%)$ eram do sexo masculino. A faixa etária prevaleceu entre pessoas acima de 50 anos (109; $87,2 \%)$.

É possível observar a prevalência de mulheres que possuem a hipertensão arterial. Este diferencial tem sido explicado pela maior percepção da mulher aos sinais e sintomas físicos e pela maior procura por assistência médica nos serviços de saúde ${ }^{10}$. Enquanto que os homens, devido a não periodicidade de exames e falta de cuidados com a própria saúde, demoram mais tempo a receber o diagnóstico da doença.

Os dados demonstram a prevalência da hipertensão na população acima de 50 anos, fato este que colabora para o aumento de comorbidades e agravos à saúde. Este fato é compatível com a história natural da HAS e com o acúmulo de fatores de risco durante a vida. Quanto mais precoces as ações de promoção à saúde maiores as chances de redução do surgimento, também precoce, das principais doenças que acometem os idosos na atualidade ${ }^{11}$.

Na relação entre longevidade e qualidade de vida, existem combinações de vários fatores, entre eles doenças biológicas e mentais, controle cognitivo, perdas de papéis ocupacionais e status social, perdas afetivas, dentre outras. Qualidade de vida, nessa idade, compreende determinantes de bem-estar tanto no campo físico quanto mental, interpessoal, espiritual e material ${ }^{12}$.

Levando-se em consideração que o conceito de qualidade de vida compreende múltiplas dimensões da vida humana e a consistência dos escores referidos pelos pacientes sobre sua qualidade de vida, os domínios do SF-36 foram analisados conforme os seguintes aspectos: estado de saúde dos indivíduos, aspectos físicos e psicossociais.

\section{Estado de saúde dos Indivíduos}

0 aspecto de estado de saúde dos indivíduos considera os domínios vitalidade e estado geral de saúde do questionário SF-36, de acordo com os escores obtidos dos 125 participantes, conforme a Tabela 1 . Ressalta-se que as médias obtidas foram analisadas em uma escala de 0 a 100, em que quanto mais próximo de zero pior a qualidade de vida, e quanto mais perto de 100 melhor a qualidade de vida.

Tabela 1- Estado geral do indivíduo e vitalidade, conforme questionário SF-36. Sobral, CE, 2019.

\begin{tabular}{lcccc}
\hline & Média & Desvio padrão & Valor Mínimo & Valor Máximo \\
\hline Estado geral de saúde & 54,52 & 17,15 & 5 & 100 \\
Vitalidade & 61,96 & 24,17 & 10 & 100 \\
\hline
\end{tabular}

Fonte: Dados da pesquisa.

0 estudo identificou a autoavaliação dos indivíduos sobre sua saúde e obteve uma média de 54,52. Essa média obtida representa um valor mediano, ou seja, os indivíduos autoavaliaram sua saúde como não sendo nem excelente e nem muito ruim, indicando que o paciente hipertenso percebe a gravidade da sua doença ${ }^{11}$. Esse fato se dá pela taxa de controle da doença e devido suas complicações que comprometem a vida cotidiana ${ }^{13}$.

$\mathrm{Na}$ atualidade, o termo saúde tem influenciado diretamente o estado de saúde e qualidade de vida das populações, grupos sociais ou indivíduo. 0 estilo de vida pode ser determinante para a apropriação dos atributos que irão favorecer a condição de saúde satisfatória ou não para o hipertenso ${ }^{14}$.

Já o domínio vitalidade é responsável por representar a energia, disposição e cansaço dos participantes. Na Tabela 1 é possível observar que o domínio vitalidade foi o que teve a maior pontuação com média de 61,96 . 
Esse fato demonstra que conviver com a hipertensão interfere na disposição, principalmente quando associado à fadiga, idade e tempo de instalação da doença ${ }^{15}$, porém não representa um fator agravante para a piora da qualidade de vida.

Com o questionário SF-36, é possível analisar o estado de saúde dos participantes de forma mais ampla. A Figura 1 explora como o participante percebia sua saúde no atual momento em comparação há um ano.

Figura 1- Percepção do estado de saúde dos participantes comparado há um ano. Sobral, CE, 2019.

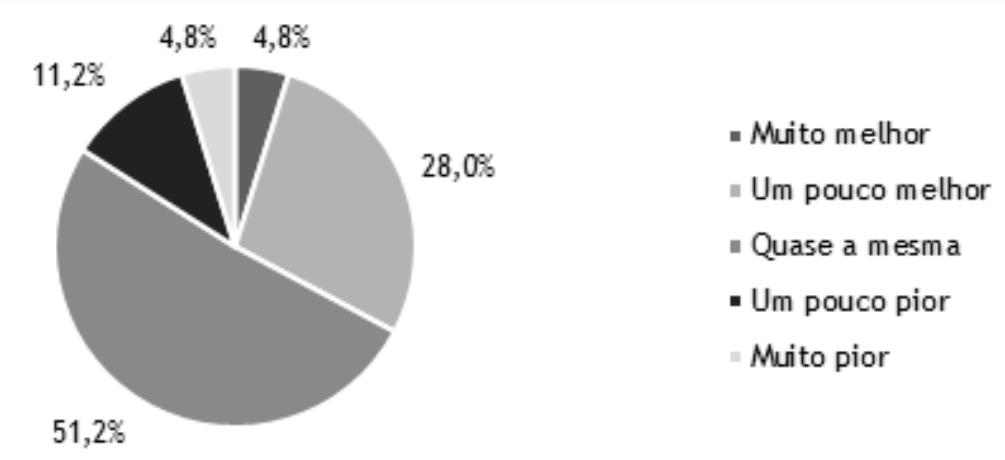

Fonte: Dados da pesquisa.

De acordo com a Figura 1, 64 participantes (51,2\%) referiram que percebem sua saúde quase a mesma há um ano, podendo se justificar provavelmente devido as manifestações clínicas relacionadas à etiologia da HAS e ao tratamento instituído, o que reflete na pouca melhoria do estado geral de saúde do participante ao longo do tempo ${ }^{16}$.

\section{Aspectos físicos}

Aqui, foram abordados os aspectos físicos que afetam a qualidade de vida das pessoas com hipertensão. Foram, então, explorados considerando os domínios dor, limitação por aspectos físicos e capacidade funcional do questionário SF-36, com o objetivo de analisar o impacto da HAS nas atividades de vida diárias e nas limitações e capacidades do corpo (Tabela 2).

Tabela 2- Dor, limitação por aspectos físicos e capacidade funcional do questionário SF-36. Sobral-CE, 2019.

\begin{tabular}{lcccc}
\hline & Média & Desvio padrão & Valor mínimo & Valor máximo \\
\hline Capacidade Funcional & 53,16 & 33,13 & 0 & 100 \\
Limitação por aspectos físicos & 45,2 & 48,86 & 0 & 100 \\
Dor & 60,66 & 31,64 & 0 & 100 \\
\hline
\end{tabular}

Fonte: Dados da pesquisa

Em relação aos domínios ou escalas do SF-36, a capacidade funcional avalia tanto a presença como a extensão das limitações relacionadas à capacidade física, a proporção que essas limitações influenciaram a vida diária do paciente e a intensidade de dor que o paciente sente ${ }^{17}$.

0 valor médio do domínio capacidade funcional foi de 53,16. Em escala de 0 a 100, é possível analisar que a hipertensão tem influência na capacidade funcional, mas não se configura como fator de risco para funções desempenhadas pelos hipertensos.

Já em relação ao domínio limitações por aspectos físicos, o valor médio foi de 45,2. Em contrapartida ao valor obtido na capacidade funcional, a presença de limitações físicas influencia de forma direta nas atividades diárias dos participantes, fator agravante para a qualidade de vida.

As limitações obtidas pela HAS incluem vertigem, cefaleia, e que devido a sua evolução rápida e, por vezes, com baixa adesão dos pacientes ao tratamento prescrito, é possível se agravar e causar limitações definitivas como AVC, doença arterial coronariana, doença arterial periférica, renal crônica e insuficiência cardíaca ${ }^{18}$. 
No domínio dor, a média obtida foi 60,66. Estudo mostra que a dor não é um sintoma que acompanha a HAS, a própria doença é considerada assintomática na grande maioria dos pacientes. A dor normalmente aparece quando HAS está associada a outra patologia ou quando apresenta uma complicação cardiovascular ${ }^{11}$.

\section{Aspectos psicossociais}

Aspectos sociais e psicológicos avaliam a integração do paciente com atividades sociais, além de verificar a ansiedade, alterações do comportamento ou descontrole emocional e bem-estar psicológico ${ }^{17}$. Considerando isso, analisou-se esse quesito a partir do questionário SF-36, apresentando como resultados o que consta na Tabela 3.

Tabela 3- Aspectos sociais, saúde mental e limitação por aspectos emocionais do questionário SF-36. Sobral, CE, 2019.

\begin{tabular}{lcccc} 
& Média & Desvio padrão & Valor mínimo & Valor máximo \\
\hline Aspectos sociais & 71,4 & 28,14 & 0 & 100 \\
Saúde mental & 66,27 & 21,78 & 0 & 100 \\
Limitação por aspectos emocionais & 48,53 & 49,26 & 0 & 100 \\
\hline
\end{tabular}

Fonte: Dados da pesquisa.

No domínio limitação por aspectos emocionais, a pontuação média foi de 48,53. A influência da HAS, nesse aspecto, dá-se em razão da necessidade de mudar os hábitos, principalmente alimentares, interferindo em atividades de lazer ${ }^{15}$.

Em aspectos sociais a pontuação média foi de 71,4. É possivel inferir que a pontuação melhorada nesse domínio possui relação com maior suporte social e familiar e que, apesar dos agravos existentes à saúde dos hipertensos, possibilitam a oferta de apoio para superação das dificuldades enfrentadas ${ }^{19}$.

0 domínio saúde mental foi a segunda maior pontuação, com média de 66,27. Médias relativamente próximas nos aspectos saúde mental e aspectos sociais demonstram que a HAS tem baixa interferência no bem-estar psicossociais dos participantes.

Diante do exposto, pode-se observar que portadores de HAS, que participaram da amostra, são influenciados por diversas limitações nas atividades de vida diária em decorrência da HAS. Quer por limitações de aspecto físico, em virtude da fadiga, cansaço e indisposição. Ou ainda, por limitações nos aspectos mentais, dada as dificuldades que o portador de HAS tem em mudar hábitos e estilo de vida para controle da pressão arterial. Contudo, possuem baixa interferência em domínios como aspectos sociais, saúde mental e dor.

Estes achados reforçam a importância dos investimentos que vêm sendo feitos, não só no Brasil, mas também no mundo, para o enfrentamento das DCNT como a hipertensão, para além do tratamento medicamentoso, visto que há consenso científico acerca dos efeitos positivos das mudanças no estilo de vida e do monitoramento frequente de fatores que interferem na HAS e na qualidade de vida ${ }^{20}$.

\section{CONCLUSÃO}

Este estudo permitiu analisar, por meio do questionário SF-36, a autopercepção dos hipertensos sobre sua qualidade de vida. Dessa forma, concluiu-se que os hipertensos percebem que sua saúde não é muito boa e a presença de limitações físicas influencia diretamente nas atividades diárias refletindo na qualidade de vida prejudicada, apesar da baixa interferência da hipertensão no bem-estar psicossocial dos participantes e nas causas de dor.

0 domínio mais comprometido entre os participantes do estudo foi limitação por aspecto físico, com média de 45,2, revelando entre os participantes que este é uma característica que atinge a qualidade de vida, por ser um fator que restringe hábitos e as atividades diárias. Já o domínio com maior média foi o de aspectos sociais $(71,4)$ demonstrando que os participantes possuem um bom suporte social e familiar, apesar da interferência da HAS em atividades de lazer, hábitos e estilo de vida.

Os resultados obtidos apontam para a necessidade eminente de mudança do olhar para a HAS, reforçando 
a importância de desenvolver estratégias de suporte e apoio na rede de atenção básica, para melhoria e ampliação do cuidado ofertado aos pacientes portadores de hipertensão. 0s achados evidenciam também a necessidade da realização de mais estudos sobre essa temática, a fim de abordar as diversas conjunturas que interferem na qualidade de vida de pacientes hipertensos e qualificar a assistência ofertada.

\section{CONTRIBUIÇÃO DAS AUTORAS}

Paloma de Vasconcelos Rodrigues contribuiu com o delineamento, análise, interpretação dos dados e redação do artigo. Maria Socorro de Araújo Dias contribuiu com a concepção, delineamento, revisão crítica do conteúdo intelectual e aprovação da versão a ser publicada. Maria da Conceição Coelho Brito contribuiu com o delineamento do artigo e revisão crítica do conteúdo intelectual. Marina Pereira Moita contribuiu com o delineamento do artigo e redação do artigo. Lielma Carla das Chagas Silva contribuiu com a concepção e o delineamento do artigo. Andrea Carvalho Araújo Moreira contribuiu com a revisão crítica do conteúdo intelectual e aprovação da versão a ser publicada.

\section{REFERÊNCIAS}

1. Rouquayrol MZ, Gurgel M. Epidemiologia e Saúde. 7 Ed. Rio De Janeiro: Medbook, 2013.

2. Weber MA, et al. Clinical practice guidelines for the management of hypertension in the community: A statement by the american society of hypertension and the international society of hypertension. J Hypertens [Internet]. 2014 [cited 2019 Feb 16];32(1):3-15. Available from: https://www.ncbi. nlm.nih.gov/pubmed/24341872.

3. Fleck MPA, Chachamovich E, Trentini C. Aplicação Da Versão Em Português Do Instrumento Abreviado De Avaliação De Qualidade De Vida "Whoqol-Bref". Rev Saúde Pública [internet]. 2000 [cited 2019 Jan 02]34:178-83. Available from: http://www.scielo. $\mathrm{br} / \mathrm{pdf} / \mathrm{rsp} / \mathrm{v34n2/1954.pdf}$.

4. Gomes DA, Bezerra EM, Silva FC, Costa TP, Ribeiro MD, Dampelo G0. Avaliação da qualidade de vida em idosos hipertensos atendidos em clínica de fisioterapia. Sanare [Internet]. 2015 [cited 2019 Mar 03];14(1):33-7. Available from: https://sanare. emnuvens.com.br/sanare/issue/view/25.

5. Araújo FNF, Figueiredo TMR, Cardoso MAA, Paes NA, Alencar HE, Santos MA. Efetividade Das Ações De Controle Da Hipertensão Arterial $\mathrm{Na}$ Atenção
Primária À Saúde. Rev Pesq Saúde [Internet]. 2016 [cited 2019 Jan 12]17(2):80-6. Available from: http://www.periodicoseletronicos.ufma.br/index. php/revistahuufma/article/view/6025.

6. Stewart SI, et al. Viper-Bp Study Investigators. More Rigorous Protocol Adherence To Intensive Structured Management Improves Blood Pressure. J Hypertens [Internet]. 2014 [cited 2019 Fev 10],32(6):1342-50. Available from: https://www. ncbi.nlm.nih.gov/pubmed/24759125

7. Gewer DM, Bandeira VA, Gelatti GT, Collet CF, Oliveria KR. Adesão ao tratamento farmacológico da hipertenso arterial na Atenção Primária. Saúde debate [Internet]. 2018 [cited 2019 Fev 23];42(116):179-80. Available from: https://www. scielosp.org/article/sdeb/2018.v42n116/179-190/.

8. IBGE. Diretoria De Pesquisas, Coordenação De População E Indicadores Sociais, Estimativas Da População Residente Com Data De Referência 10 De Julho De 2018. 2018. Disponivel em:< https:// cidades.ibge.gov.br/brasil/ce/sobral/panorama>.

9. Ciconelli RM. Tradução Para 0 Português $E$ Validação Do Questionário Genérico De Avaliação De Qualidade De Vida "Medical Outcomes Study 36-Item Short-Form Health Survey (Sf-36)" [Tese]. São Paulo: Universidade Federal De São Paulo; 1997.

10. Zangirolani LM, Assumpção D, Medeiros MA, Barros MA. Hipertensão arterial autorreferida em adultos residentes em Campinas, São Paulo, Brasil: prevalência, fatores associados e práticas de controle em estudo de base populacional. Ciên. Saúde Colet [Internet]. 2018 [cited 2019 Feb 12];23(4):1221-32. Available from: https://www.scielosp.org/article/ csc/2018.v23n4/1221-1232/.

11. Francisco PM, Segri NJ, Borim FS, Malta D.C. Prevalência simultânea de hipertensão e diabetes em idosos brasileiros: desigualdades individuais e contextuais. Ciência \& Saúde Coletiva [Internet]. 2018 [cited 2019 Jan 02]23(11):3829-40. Available from: http://www.scielo.br/scielo.php?script=sci abstract \& pid $=$ S1413-81232018001103829\&lng $=p t \& n$ rm=iso.

12. Silva CC, et al. Condições de saúde e qualidade de vida de idosos participantes de um programa de responsabilidade social. Rev. Aten. Saúde. [Internet]. 2017 [cited 2019 Feb 24];15(52):15-20. Available from: http://seer.uscs.edu.br/index.php/ revista ciencias saud

13. Dantas RC, Roncalli RA. Protocolo para indivíduos hipertensos assistidos na Atenção Básica em Saúde. Ciênc saúde colet. [Internet]. 2019 [cited 2019 Feb 25];24(1):295-306. Available from: https://www. scielosp.org/article/csc/2019.v24n1/295-306/.

14. Silva RAR, Sakon P0. Auto percepção do estado de 
saúde de hipertensos. Rev enferm UFPE [Internet]. 2018 [cited 2019 Jan 21];12(7):1826-34. Available from: $\quad$ https://pesquisa.bvsalud.org/portal/ resource/pt/biblio-986525.

15. Scala LC, Magalhães LB, Machado A. Epidemiologia Da Hipertensão Arterial Sistêmica. In: Moreira Sm, Paola Av; Sociedade Brasileira De Cardiologia. Livro Texto Da Sociedade Brasileira De Cardiologia. $2^{\mathrm{a}}$. Ed. São Paulo: Manole; 2015.p.780-5.

16. Brito DMS, Araújo TL, Galvão MT, Moreira MT, Lopes MV. Qualidade de vida e percepção da doença entre portadores de hipertensão arterial. Cad Saúde Pública [Internet]. 2014. [cited 2019 Jan 02];24(4):933-40. Available from: http://www. scielo.br/scielo.php?script $=$ sci arttext \&pid=S0102311X2008000400025.

17. Suzano DS, Almeida MC, Massa LD, Wegred M. A importância da qualidade de vida em pacientes hipertensos. Saúde em redes [Internet]. 2016 [cited 2019 Feb 03];2(1): 53-63. Available from: http:// revista.redeunida.org.br/ojs/index.php/rede-unida/ article/view/660.

18. Sociedade Brasileira de Hipertensão. $7^{\text {a }}$ diretriz brasileira de Hipertensão Arterial. Brasilian Journal Hypertension [Internet]. 2017 [cited 2019 Jan 16];24(1):1-91. Available from: http:// departamentos.cardiol.br/sbc-dha/profissional/ revista/24-1.pdf.

19. Brasil. Ministério Da Saúde. Secretaria De Atenção À Saúde. Departamento Da Atenção Básica. Política Nacional Da Atenção Básica. Brasília: Ministério Da Saúde. 2014.

20. Nunes LC, Santos CA, Serra MA. Fatores de risco e cuidados de enfermagem ao idoso hipertenso: revisão integrativa. Revista Sanare [Internet]. 2014 [cited 2019 Jan 13];13(2). Available from: https:// sanare.emnuvens.com.br/sanare/article/view/581.

21. Malta DC, Silva Júnior JB. 0 plano de ações estratégicas para o enfrentamento das doenças crônicas não transmissiveis no brasil e a definição das metas globais para o enfrentamento dessas doenças até 2025: uma revisão. Epidemiol Serv Saúde [Internet]. 2014 [cited 2019 Jan 25];22(1):151-64. Available from: http://scielo. iec.gov.br/scielo.php?script=sci arttext\&pid $=$ S1679-49742013000100016.

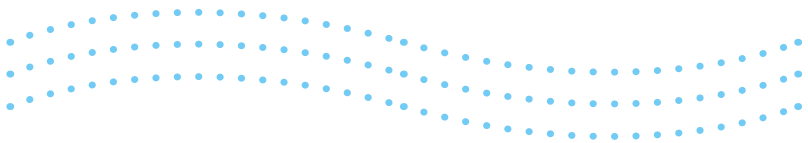

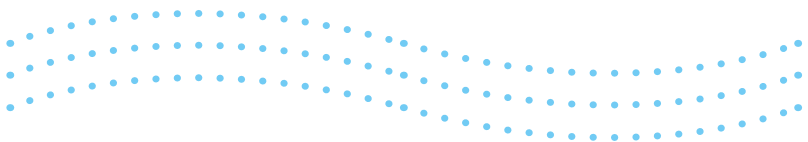
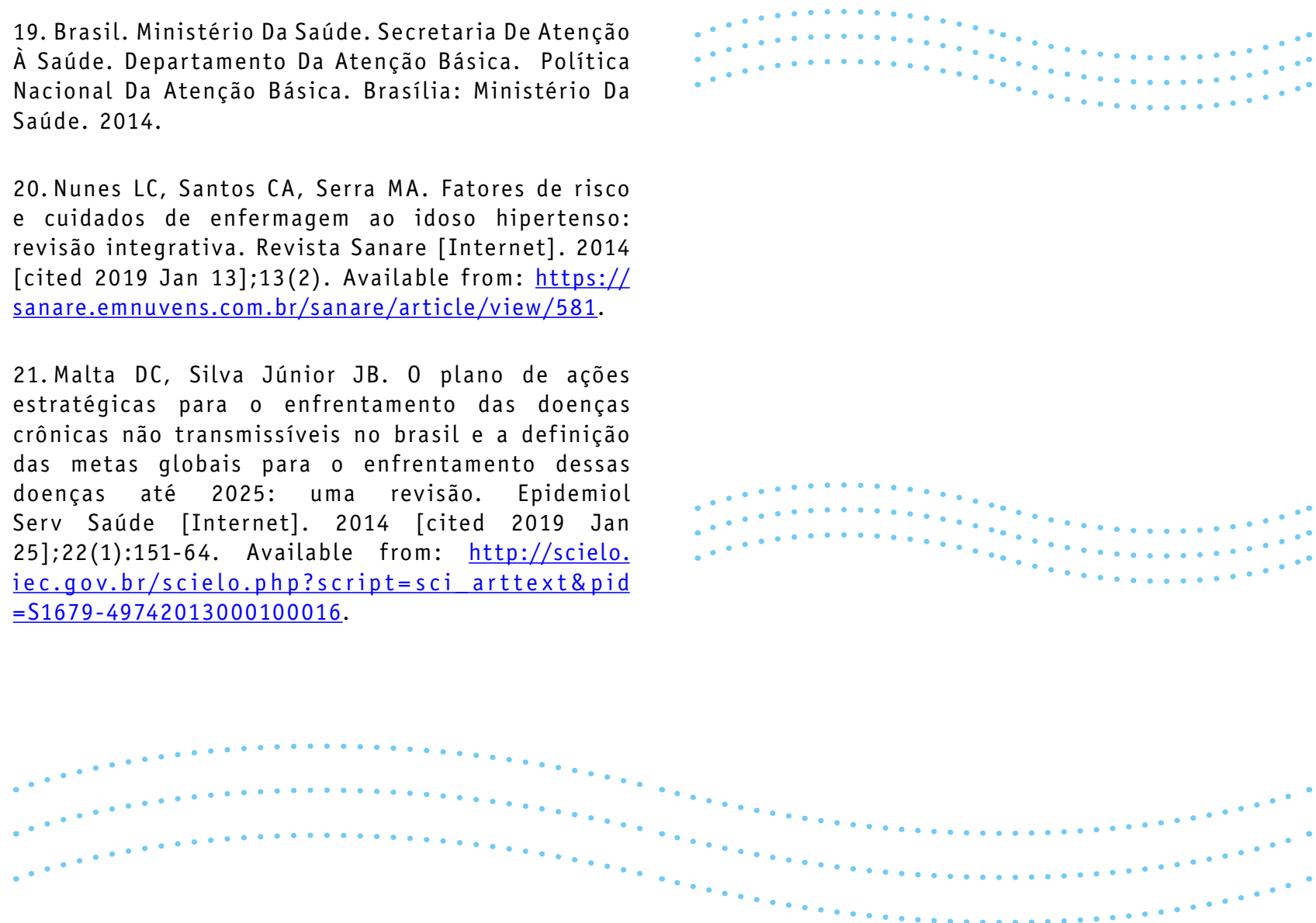